\title{
A technique for the study of spatiotemporal aspects of paw contact patterns, applied to rats treated with a TRH analogue
}

\author{
K.A. CLARKE \\ University of Sheffield, Western Bank, Sheffield, England
}

\begin{abstract}
The analysis of footfall patterns can help in the evaluation of normal and disturbed neurological function. Previous methods, however, such as inking the feet, give only a snapshot of maximum contact without temporal information. This paper describes the use of an alternative technique, with which these limitations can be overcome through the use of light reflected within a glass plate to illuminate paw contact areas during the stance phase of locomotion. Computer analysis demonstrates no difference in maximum contact area between forepaws and hindpaws, with the major contact structures being pads DP2 and DP3, but there are numerous differences in deployment. For example, the forepaw deploys tactile sensory-rich structures early in the contact cycle. The effects of TRH analogue CG3703 on aspects of hindpaw contact demonstrate that treated rats make more extensive use of distal parts of the paw. The results are discussed in terms of CG3703-induced postural changes.
\end{abstract}

The analysis of locomotor activity is a valuable tool in neuropharmacological, neurophysiological, and behavioral studies. Although the majority of experiments have quantified such activity (Andrews \& Sahgal, 1983; Beninger, Cooper, \& Mazurski, 1985), valuable information is contained in how, rather than how much, an animal moves. Gait analysis includes a number of approaches, such as the study of the interplay of forward velocity with stride time and length, and examinations of relationships between the component variables of stride such as stance and swing times (Clarke \& Parker, 1986; Hruska, Kennedy, \& Silbergeld, 1979).

One neglected aspect of gait study, the analysis of footfall patterns, can, however, yield valuable data. For example, Schaefer and Kretsch (1987) showed footprint examination to be a sensitive index of the neural consequences of Vitamin B6 deficiency, and De Medinaceli, Freed, and Wyatt (1982) utilized footprint examination in the evaluation of sciatic nerve recovery following peripheral nerve injury. An existing technique, inking the feet, disrupts the animal's behavior and provides only a distorted snapshot of maximal contact. Neither does it permit the study of any changes in the contact pattern that might occur during this phase. In this paper, I describe an alternative method whereby a freely moving rat produces footfall data that retain temporal information, lend themselves to computer analysis, and may be applicable to other aspects of footfall such as pressure distribution. The technique is demonstrated in two ways. First, to permit examination

Correspondence should be addressed to K. A. Clarke, Department of Biomedical Science, University of Sheffield, Western Bank, Sheffield S10 2TN, England. of forepaw and hindpaw footfall patterns during walking gait in the rat. Second, to assess its sensitivity to neuropharmacological disturbance of locomotion. These rats were given CG3703 (6-methyl-5-oxo-thiomorpholinyl-3 carbonyl-His-Pro- $\mathrm{NH}_{2}$, kindly supplied by Grunenthal $\mathrm{GmbH}$, Anachem), an analogue of TRH producing postural and locomotor changes (Andrews \& Sahgal, 1988; Clarke \& Steadman, 1989).

\section{METHOD}

Paw contact analysis was undertaken in 6 neurologically normal and 6 CG3703-treated female Wistar rats in the weight range of 190-210 g. The latter received an i.p. injection of 5-mg/kg CG3703 dissolved in $0.5 \mathrm{ml}$ of $0.9 \%$ saline and were locomotor tested $15 \mathrm{~min}$ later. Prior to experimentation, the rats were kept 3 to a cage, on a light:dark cycle of 12:12 h with light on at 8 a.m.; food and water were allowed ad lib. All experiments were performed between 12 noon and 4 p.m. to minimize any effects of diurnal variability.

The apparatus consisted of a Plexiglas chamber with internal dimensions of $100 \times 10 \times 10 \mathrm{~cm}$ housed in a dedicated, soundproofed behavioral laboratory (Figure 1). A mirror placed at an angle of $45^{\circ}$ below the central $30-\mathrm{cm}$ section of the chamber allowed floor/paw contacts to be viewed (Clarke \& Steadman, 1989). To make data comparable, it is important to know the velocity and stride time of the rat, which indicate whether or not it is using a walking gait. Since only the parts of the rat close to the air/glass interface were consistently visible to the camera (see below), it was not possible to calculate velocity directly from the video image. Therefore, pairs of infrared transmitter/receivers at each end of the central section were employed to generate voltage pulses when a rat broke the beams, permitting calculation of the rat's velocity over this section. Stride time was calculated from successive placements of the same paw. Contact points between the paw and floor were transduced by adapting the method of Betts and Duckworth (1978), which was originally applied to measure plantar pressures produced by the human foot. The base of the chamber was made of 6-mm glass. Light was shone into one of the long edges from an 8-W, white 


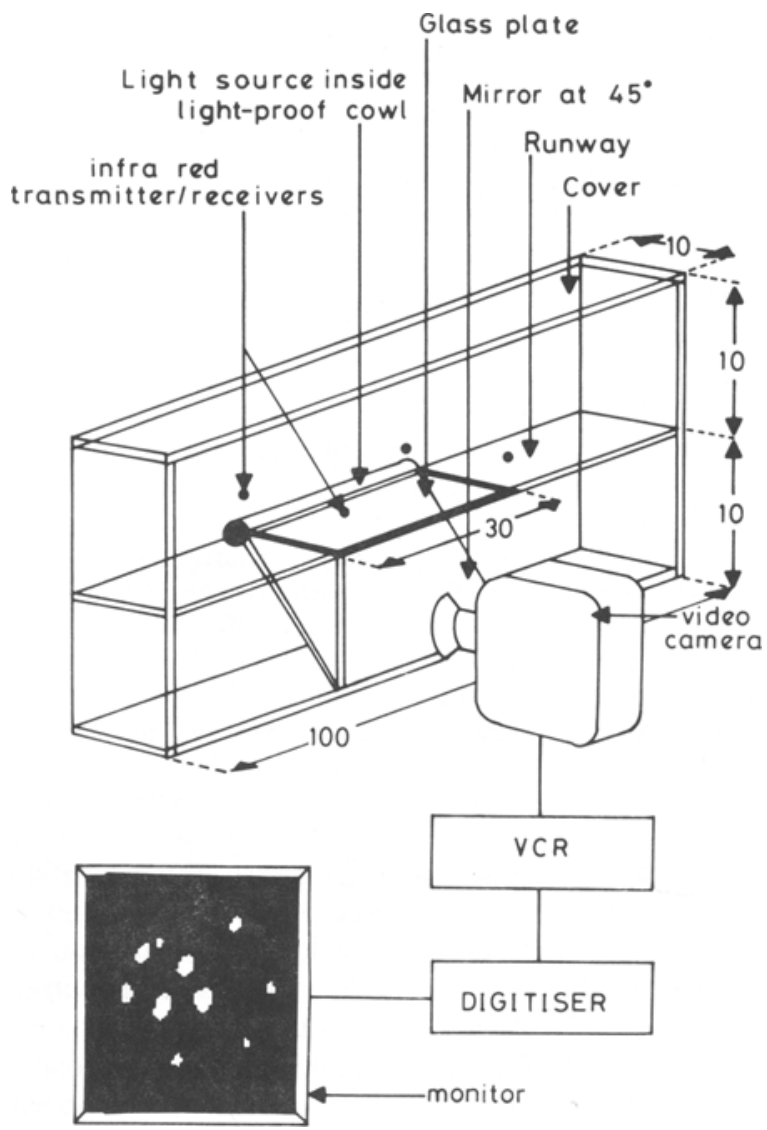

Figure 1. Apparatus used for collection and analysis of fontfall patterns.

fluorescent tube (Thorn, UK). The room was dark, and the light tube was cowled to prevent light escaping elsewhere. Light entering the glass edge was internally reflected between the top and bottom surfaces until the paw made contact with the glass, scattering light at the paw/glass boundary. Thus, paw contact points were brightly illuminated areas (Figure 1).

Each rat moved about the chamber individually, and its footfall patterns during locomotion were viewed by a video camera (JVC GX-98E, 25 fields/sec). The image, and the voltage pulses needed to calculate velocity, were transmitted to another laboratory where the video image was recorded on tape (Fuji HG E180) on a Panasonic G12 Super Still recorder for further analysis. Successive video frames during paw contact cycles were captured and stored in a video digitizer (Image $256 \times 256$ pixels per frame, 64 gray levels per pixel, Eltime Vision Systems, U.K.) incorporated into a Viglen Series $1640 \mathrm{~K}$ personal computer. Dedicated software (Ramases 3, Eltime Video Systems) analyzed the stored images in terms of the distribution and relative areas of each of the discrete contact points. The system was calibrated by imaging areas of graph paper placed on the glass floor.

Stance time (the time that the paw is in ground contact) varies with stride time, and therefore the number of frames captured during the contact phase ranged from 5 to 12 . To pool data, contact periods were normalized to five phases: start, early, middle, late, and end. The start and end phases contained the first and last frames, respectively. The remaining frames were allocated equally to the early, middle, and late periods. Any remaining frames were placed in the middle period.

\section{RESULTS}

\section{Neurologically Normal Rats}

Ten forepaw and ten hindpaw stance times gained from 6 neurologically normal rats (maximum 2 forepaw and 2 hindpaw stances per rat) were analyzed within the range of walking velocities of $10-50 \mathrm{~cm} / \mathrm{sec}$ and stride times of 200-1,000 msec (Clarke \& Parker, 1986).

\section{Contact Points}

Contact points for the forepaw were the four fleshy digital tips, D1-D4, the three distally placed pads at the bases of the digits, DP1-DP3, the pollex (rudimentary thumb), Po, and two more proximally placed pads, PP1-PP2 (Figures 2 and 3 ).

Contact points for the hindpaw were the five fleshy digital tips, D1-D5, the four pads at the bases of the digits, DP1-DP4, and the two more proximally placed pads, PP1-PP2 (Figures 2 and 3).

Total contact area was maximal for the forepaw during the middle phase and reached a maximum for the hindpaw during the early phase. Maximal contact areas for forepaw and hindpaw were not significantly different at $27.6 \pm 3.7$ and $28.6 \pm 4.8 \mathrm{~mm}^{2}(M \pm 1 S E M)$, respectively.

For both forepaw and hindpaw, the most extensive ground contact was with Pads DP2 and DP3. These structures accounted for $42 \%-50 \%$ of the total contact area for the forepaw, for which they were maximal in the late phase, and for $41 \%-68 \%$ of the total contact area for the hindpaw, for which they were maximal in the middle of the cycle. Transition between dual stance (with both forepaws and both hindpaws in ground contact) and single stance (only paw under observation in ground contact) $o c-$

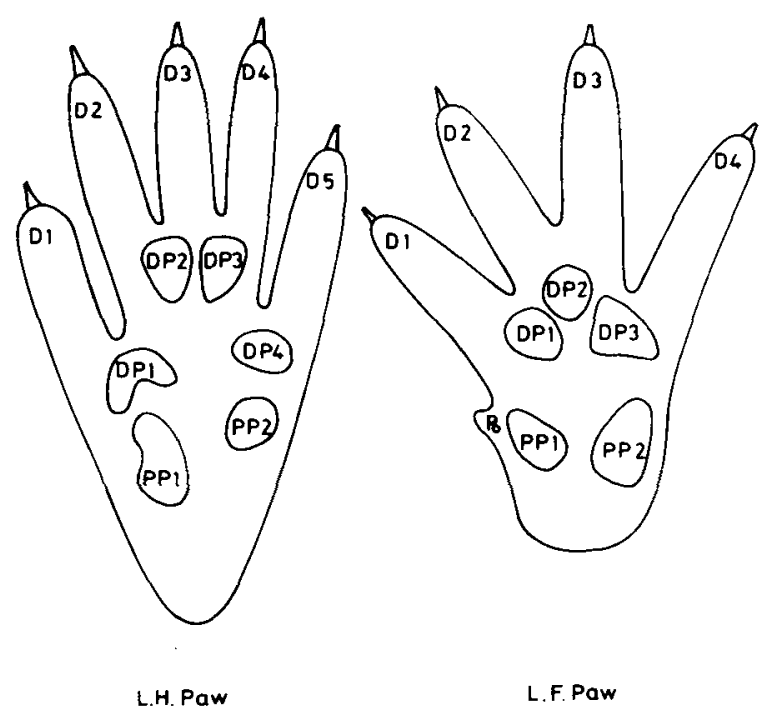

Figure 2. Diagrams of palmar surface of left hindpaw and forepaw. D1-D5, digits; DP1-DP4, distal pads; Po, pollex; PP1-PP2, proximal pads. 


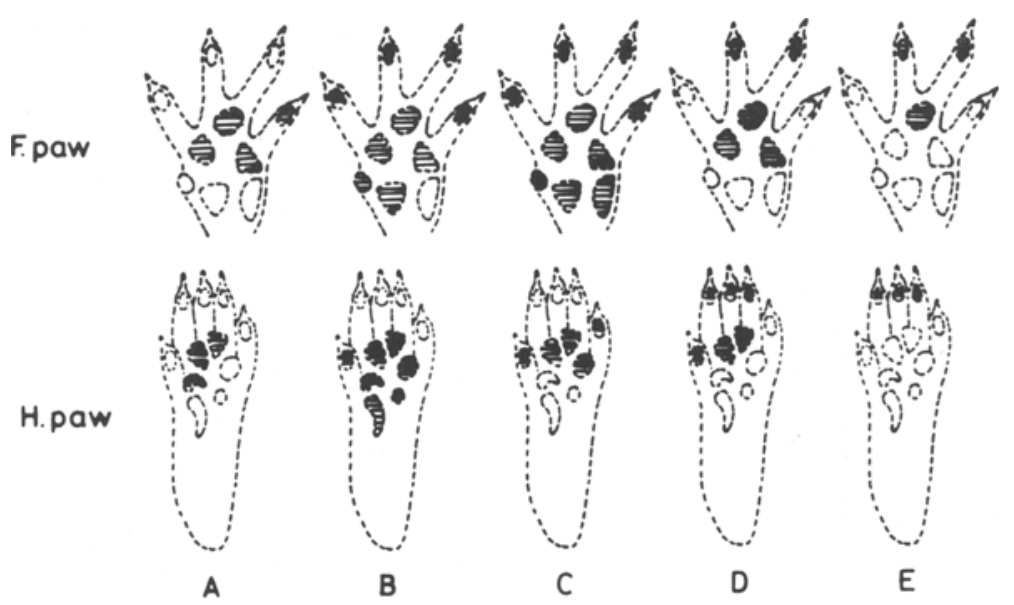

Figure 3. Diagrams A-E summarize typical paw contact points (shaded) for left forepaw (upper) and hindpaw (lower) at the five phases of contact from (A) start to (E) end.

curred within plus or minus one frame of the end of the early phase and the beginning of the middle phase.

\section{Contact Sequences}

Forepaw contact cycle starts with the distolateral structures D4, DP1, DP2, and DP3 (Figure 4, S column, Zones $B$ and $C$ ). Downward displacement and/or medial rotation of the paw during the early phase brings contact with other distal (D1, D2, and D3; $\mathrm{E}_{1}$ column, Zone A)

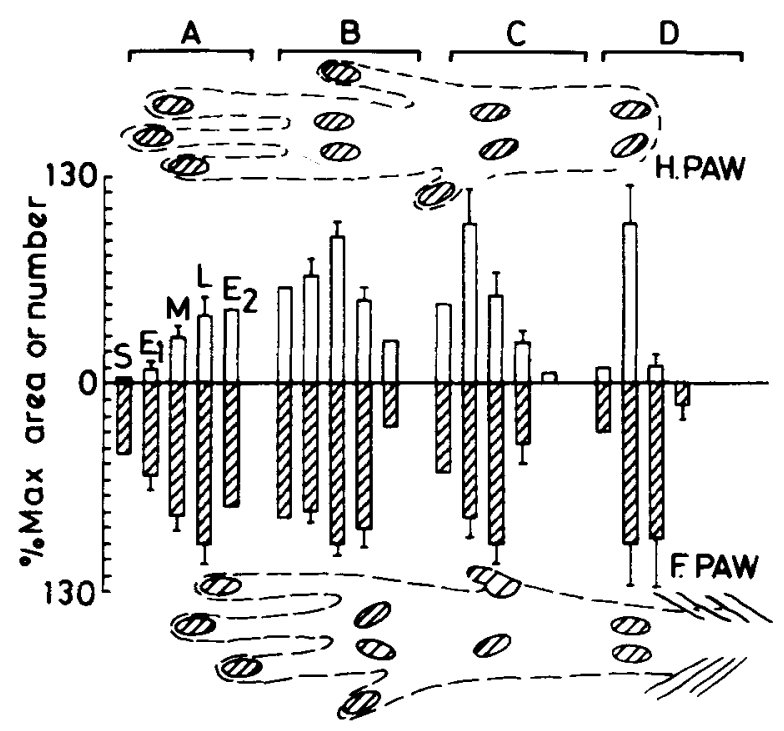

Figure 4. Histograms lllustrating the extent of hindpaw (open columns) or forepaw (shaded columns) contact for each of the five phases of stance (S, start; $E_{1}$, eariy; $M$, middle; $L$, Late; $E_{2}$, end). The paw diagrams have been extended to separate more clearly the possible contact points into a distoproximal sequence of four zones, A-D. Each column is the mean percent maximum number of instances of contact (columns $S$ and $E_{2}$ of each zone), or mean (+1 SEM) percent maximum area of contact of that zone (columns $E_{1}, M$ and $\mathbf{L}$ ). and proximomedial (Po and PP1; $\mathrm{E}_{1}$ column, Zones C and D) structures. Analysis of the deployment of PP1 shows that the first part of it to contact the surface is its distal edge. The middle phase involves further downward displacement resulting in maximal multipoint contact with all contact zones of the paw surface ( $M$ column, Zones A, B, C, and D). The late phase displays a slightly medially directed movement such that contact with D4 is lost before D1, D2, and D3. The contact cycle ends with a 3-point contact of D2, D3, and DP2 ( $E_{2}$ column, Zones $A$ and $B)$. The hindpaw contact cycle starts with the distal structures DP1, DP2, and DP3 (S column, Zones B and $C$ ) with little or no digital contact ( $S$ column, Zone A) (Figure 4). Downward displacement during the early phase brings in proximal (PP1 and PP2; $\mathrm{E}_{1}$ column, Zone D) and lateral (DP4, PP2; $E_{1}$ column, Zones $C$ and D) structures. Of the digits, only D1 makes extensive contact at this time. During the middle phase, there is no significant change in contact area, but elevation of the ankle causes the paw to roll up, shifting contact to more distal structures (DP2, DP3) and now including D5 (M column, Zone B). The late phase brings maximal contact for the most distal structures, the tips of D2, D3, and D4, as the paw rolls further, and ends in contact with these structures, particularly D3 (columns $L$ and $E_{2}$, Zone A). In the majority of steps during walking, the hindpaw is placed behind the previous forepaw position.

\section{CG3703-Treated Rats}

A modified $t$ test showed that stance time was significantly reduced from $442 \pm 20 \mathrm{msec}$ in controls to $360 \pm 20 \mathrm{msec}$ in treated rats $(M \pm 1 S E M, n=20, p<$ .006). Deployment of the most distal hindpaw contact zone (Zone A) has been analyzed for 10 stance times in 6 CG3703-treated rats (maximum 2 stances per rat). Figure 5 (which also incorporates equivalent control data from Figure 4) illustrates that, at any particular contact phase, the CG3703-treated rat makes more extensive use of these distal contact zones than do the control rats. 


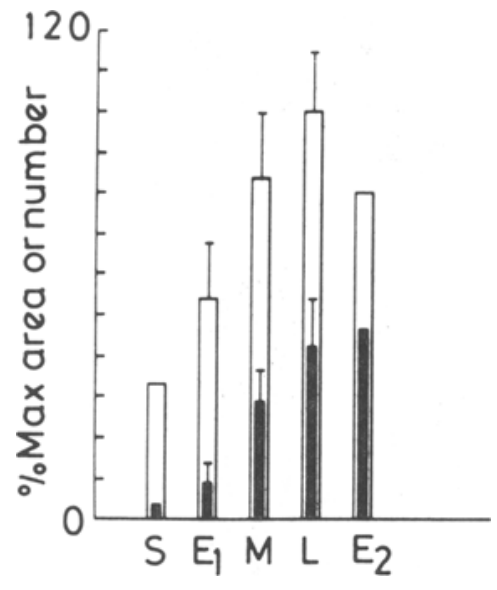

Figure 5. Histograms illustrating the extent of hindpaw contact of Zone A (D2, D3, D4) for each of the five phases of stance, of control (shaded columns, data repeated from Figure 4), and CG3703treated (open columns) rats. The phases are represented as $S$, start; $E_{1}$, early; $M$, middle; $L$, late; and $E_{2}$, end. Each column is the mean percent maximum number of instances of contact (columns $S$ and $E_{2}$ ), or mean (+1SEM) percent maximum area of contact (columns $E_{1}, M$, and $L$ ).

\section{DISCUSSION}

The forepaw contact cycle starts with distolateral structures (D4, DP1, DP2, and DP3) that may be a consequence of an outward limb swing during weight shifting from the opposite side. Transition between dual and single stance time occurred within plus or minus one frame of the end of the early and the beginning of the middle phases. This is broadly equivalent to the change from soft to hard stance found by Cohen and Gans (1975)-that is, the shift from soft contact to maximum weight bearing. Downward displacement and/or medial rotation of the forepaw during the early phase brings contact with various distal structures (D1, D2, and D3) and maximal contact with proximomedial structures (Po and PP1). This is particularly interesting during what is essentially the soft contact phase.

Previous neurophysiological investigations have shown that most of these structures (tips of D2 and D3, DP1, Po, and PP1) are particularly well represented in the ventrobasal thalamus, in terms of the number of neurons per unit surface area of skin (Angel \& Clarke, 1975). This region of the thalamus processes tactile information for tactile guided movement (Angel \& Clarke, 1975). Further analysis of the deployment of PP1 shows that the first part of it to contact the surface is its distal edge. Again, previous work has shown that many of the ventrobasal neurons representing the skin along this edge have small receptive fields (Angel \& Clarke, 1975). I suggest that these sensory-rich structures are presented to the surface early, during the soft contact phase, to sample the suitability of the surface and assist the programming of the rest of the stance phase. The late phase displays a slightly medially directed wave of contact loss. This might be associated with a medially directed thrust to shift weight to the opposite side.

Although, from total contact area, DP2 and DP3 are the most important structures, previous work has shown that their tactile sensibility is poorly represented in the ventrobasal thalamus. The sensory-poor DP2 and DP3 may therefore be deployed as the major weighttransmitting structures for the forepaw during the middle and later parts of the contact cycle.

Tactile somatotopic ventrobasal representations of the hindpaw's surface structures are much poorer than those of the forepaw (Angel \& Clarke, 1975). I suggest that the hindpaw's tactile exploratory uses during locomotion are minimal, and that it is used primarily as a weightbearing, thrust-producing structure. Hruska et al.'s (1979) analysis of footprints by rats locomoting over a range of gaits suggested that the hindpaw substantially overlapped previous forepaw placement. Although this attractive proposition would allow for tactile forepaw exploration of the surface prior to placement of the hindpaw in the same place, present observations at walking velocities indicate that, in the majority of steps taken, the hindpaw was placed behind the previous forepaw position.

A number of workers have observed that TRH and its analogues cause profound changes in the posture and locomotion of rats. These include the elevation of the hindquarters with reductions in stance time, and increases in swing time during locomotion (Andrews \& Sahgal, 1983; Clarke \& Steadman, 1989). The reductions in stance time are confirmed by the present work, which also shows that these postural and locomotor changes are accompanied by alteration in the paw contact pattern deployed to sustain the stance phase. Further work is required to determine whether all contact zones are increased, or whether there is a relative shift from proximal to distal zones.

This technique has previously been applied in humans to determine pressure distributions during footfall (Betts $\&$ Duckworth, 1978). However, to achieve pressure transduction, a suitably deformable interface needs to lie between the paw and glass. We have not yet found a material that gives sufficient resolution for the lower pressures of the rat paw. An appropriate choice of interface material would allow different aspects of the contact cycle to be studied.

In conclusion, this technique is useful in the evaluation of normal and disturbed locomotion, and it can be applied in a variety of behavioral studies.

\section{REFERENCES}

ANDREWS, J. S., \& SAHGAL, A. (1983). Effects of thyrotropin releasing hormone, metabolites and analogues on locomotor activity in rats. Regulatory Peptides, 7, 97-109.

Angel, A., \& Clarke, K. A. (1975). An analysis of the representation of the forelimb in the ventrobasal thalamic complex of the albino rat. Journal of Physiology, 249, 399-423. 
Beninger, R. J., CoOper, T. A., \& Mazurski, E. J. (1985). Automating the measurement of locomotor activity. Neurobehavioral Toxicology \& Teratology, 7, 79-85.

BetTs, R. P., \& DUckworTh, T. (1978). A device for measuring plantar pressures under the sole of the foot. Engineering in Medicine, 7, 223-228.

Clarke, K. A., \& Parker, A. J. (1986). A quantitative study of normal locomotion in the rat. Physiology \& Behavior, 38, 345-351.

Clarke, K. A., \& Steadman, P. (1989). Abnormal locomotion in the rat after administration of a TRH analogue. Neuropeptides, 14, 65-70.

COHEN, A. H., \& GANS, C. (1975). Muscle activity in rat locomotion: Movement analysis and electromyography of the flexors and extensors of the elbow. Journal of Morphology, 146, 177-196.
De Medinaceli, L., Freed, W. J., \& Wyatt, R. J. (1982). An index of the functional condition of rat sciatic nerve based on measurements made from walking tracks. Experimental Neurology, 77, 634-643.

Hruska, R. E., Kennedy, S., \& Silbergeld, E. K. (1979). Quantitative aspects of normal locomotion in rats. Life Science, 25, 171-180.

SCHAEFER, M. C., \& KRETSCH, M. J. (1987). Quantitative assessment of motor and sensory function in Vitamin B6 deficient rats. Nutrition Research, 7, 851-863.

(Manuscript received June 18, 1991; revision accepted for publication February 18, 1992.) 